\title{
Design of Distributed Control System Software Using Client-Server Architecture
}

\author{
Yih-Ping Luh, Shean-Shyong Chiou, Jau-Woie Chang \\ Department of Mechanical Engineering \\ National Taiwan University, Taipei, Taiwan, China
}

\begin{abstract}
In the paper, we adopt the client/server architecture in designing a distributed control system software. In industrial automation, each application can be thought as a combination of many server and client processes. For example, a data acquisition system is several data collecting processes (the clients) plus several common server processes such as the IO server processes. These distributed processes cooperate their own functionality to achieve the system-wide tasks. In the client/server architecture, we reduce the software complexity and increase the software reusability of a large control system.
\end{abstract}

\section{INTRODUCTION}

The design philosophy of a traditional control software has been set to include all the required functionality in one package. This philosophy works well when the system is relatively small. However, as the complexity of a system increase, the complexity of the control software will increase exponentially fast [1]. Therefore, a client-server architecture must be used to reduce the software complexity. It is also reported that the client/server technology can make it easier for users to access information, to use and to develop applications, and to manage a distributed computing environment [2].

In addition to control system software, we introduce the concept of the control system architecture. Different architecture has different system characteristics [3]. A complete system and software architecture are given in this paper.

The rest of the paper is organized as follows: Section II briefly states some design issues of a control system. Section III introduces our implementation environment. Finally, some concluding remarks are discussed in section IV.

\section{CONIROL SYSTEM}

A complete control system has to include some functions such as data IO, monitoring, alarming, computing, data acquisition, etc. A large system is developed using highly modular and expandable software architecture[1]. All control functions are implemented independently from each other. In the paper, we put some related functions together within one control node. Each control node should achieve its preset functionality. However, there is another issue about joining these control nodes together to accomplish system level tasks.

"Control architecture makes a control system from control components [3]." The control architecture determines the stability, structure and ways of communication among control nodes. More details about control architecture have been described in several papers where the reader is referred [3-5] and the references therein In the paper, we pick the heterarchical form as the control architecture. The main advantages of heterarchical control architecture are reducing software complexity, reducing coupling among modules, and implicit fault-tolerance.

As the size of a control system increase, the complexity of the control software increases exponentially fast. Therefore, find a way to reduce the complexity becomes important. "Modularity" is the answer to the problem [1]. In this paper, we propose a client/server technology in designing control software. The client/sever technology has the feature of modularity and other benefits such as: lower cost, higher productivity, longer system life cycle, and better software reusability. The client/server architecture is an approach to software where the client asks for and receives services from the server. These client and server applications are executed independently from each other.

\section{IMPLEMENTATION}

\section{A. System Architecture}




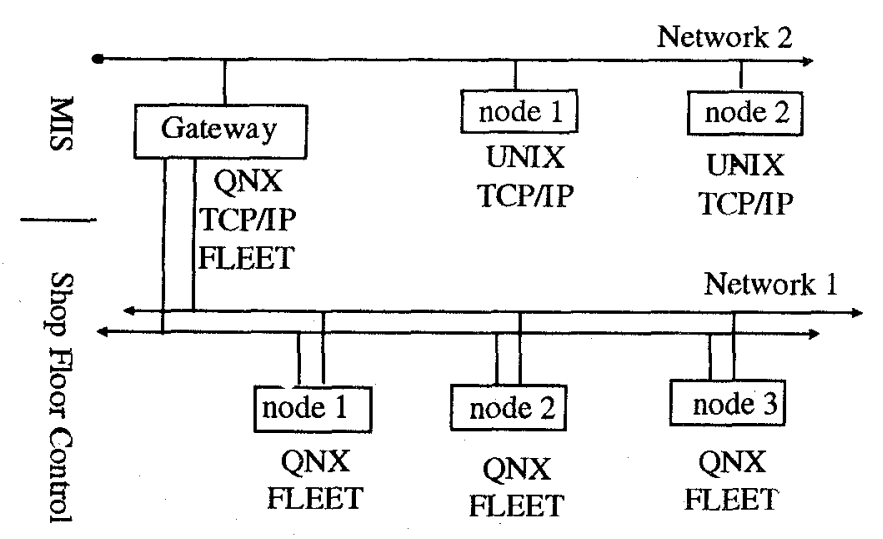

Fig. 1 system architecture

Our system topology is described in Fig. 1. The network 1 is the shop floor control level and the network 2 is the MIS (Management Information System) level. Each node in the network 1 uses the QNX as its operating system and dual network path for communication purpose. The QNX operating system is a distributed real time operating system $[6,7]$. The FLEET (Fault-Tolerant, Load-balancing, Efficient, Extensible, Transparent) networking technology in QNX makes the application more reliable and efficient. When there is more than one path used for communication, the QNX transmits data over both network paths simultaneously. Also, if any one path is failure, it will reroute all data through another path.

In the MIS level, we build our network application in the TCP/IP network environment with socket library. The socket library is available on a lot of platforms, such as UNIX BSD socket, Windows socket, etc. With the socket library, we can

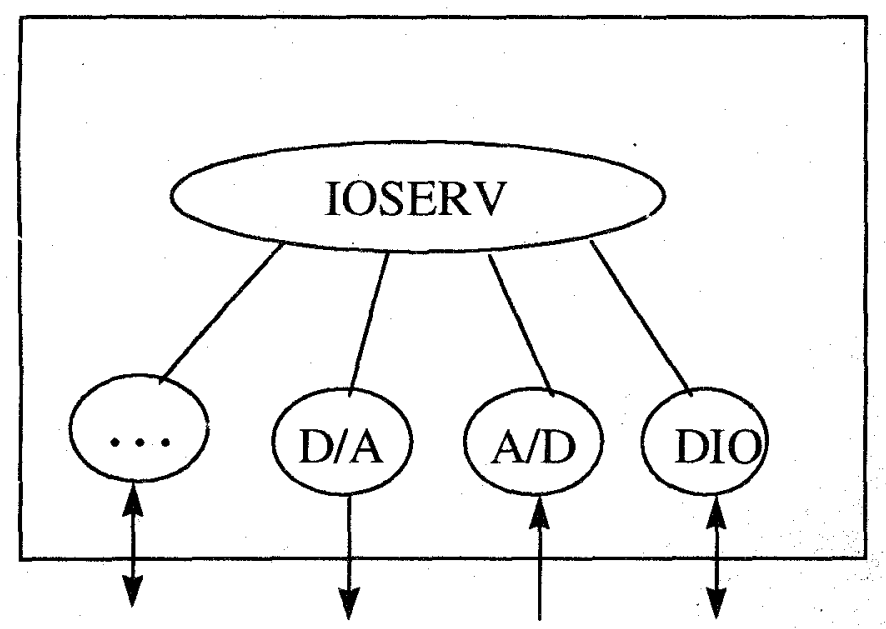

Fig. 2 IO node software configuration

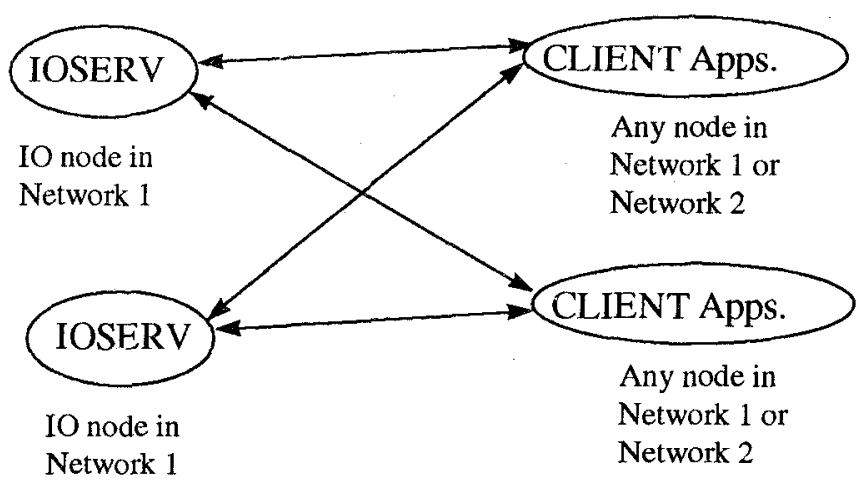

Fig. 3 Relationship between client applications and IOSERV processes

easily transfer our application to another operating system. In the paper, we adopt the UNIX as our operating system in network 2.

\section{B. Software Architecture}

We design our control software in a client/sever approach. As described in Fig. 2, there are one IOSERV process and many low level physical IO processes in each IO node located in network 1 . The IOSERV process maintains a real time IO point data table and takes the responsibility of client requests. Any client IO request is sent to the IOSERV process within each IO node. The real time IO point data table is updated by low level physical IO processes. In the software environment, we can develop our client application independently from IOSERV process. As depicted in Fig. 3, the client application can only access these IO points through IOSERV processes, and all client/server processes are independent from each other. Compared to the traditional method which application may access IO points directly, this method not only isolates the application from hardware interface, but also simplified the application program. As a result, any time we need to modify the client/server application process, it is not necessary to modify other processes because of client/server independence.

As shown in Fig. 4, there are two IOSERV processes, relative to $\mathrm{A} / \mathrm{D}$ processes and PLC driver process respectively. In addition, the alarm system must have one client application associated with alarming, also the monitoring system must have one client application associate 


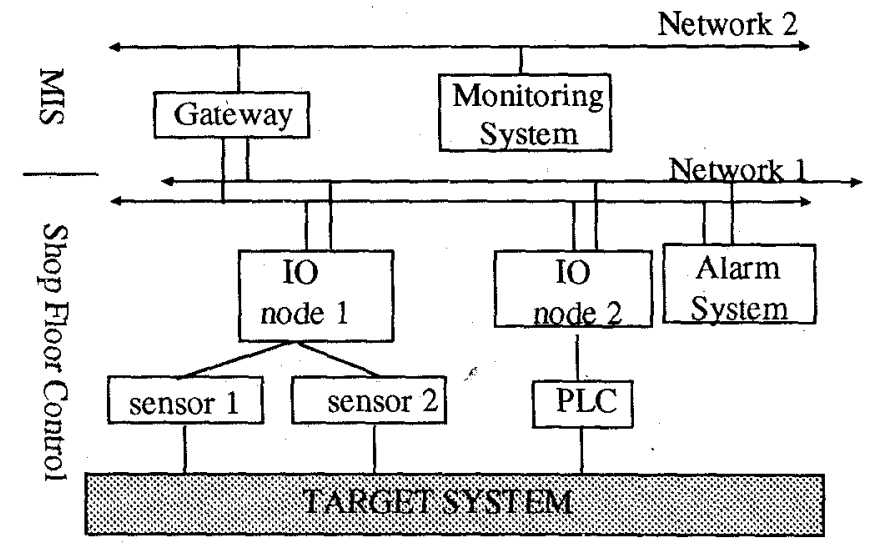

Fig. 4 An application example

with data monitoring. Both systems need to access IO point data though two common IOSERV processes. Whenever we need to update either of these two systems, it does not need to change another system at all. The monitoring system is built on the UNIX operating system using TCP/IP socket programming. The alarming system is built on the $\mathrm{PC} / \mathrm{QNX}$ environment through the Inter-Process Communication.

\section{CONCLUSION}

This paper has outlined some considerations in the software design of distributed control system. The client/server software architecture not only reduces the complexity of control software design, but also makes applications independent from each other. This independence makes the software maintenance easier. We also give an application example in the paper. With the growing needs of the control system software, further research about the client/server application is certainly necessary.

\section{REFERENCES}

[1] Maarten Boasson, "Control System Software," IEEE Trans. on Automatic Control, Vol. 38, No. 7, Jul. 1993, pp.1094-1106.

[2] JrJung Lyu, "On Developing an Inventory Management System in the Client/Server Environment," Computers and Industrial Engineering, Vol. 29, No. 1-4, 1995, pp.93-97.

[3] M. Dilts, N.P. Boyd, H.H. Whorms, "The Evolution of Control Aritechtures for Automated Manufacturing Systems," Journal of Manufacturing Systems, Vol. 10, No. 1, pp. 79-93.

[4] . Thomas J. Crowe, Edward J. Stahlman, "A proposed structure for distributed shopfloor control," Integrated Manufacturing Systems, Vol. 6, No. 6, 1995, pp.31-36.

[5] N.A. .Duffie, R.S. Piper, "Nonhierarchical control of manufacturing systems," Journal of Manufacturing Systems, Vol. 5, No. 2, 1986, pp. 137-139.

[6] Dan Hildebrand, "An Architectural Overview of QNX," Proceeding of the Usenix Workshop on Micro-Kernel \& Other Kernel Architectures, Seattle, April 1992.

[7] QNX System Architecture, Quantum System Ltd., 1992. 